\title{
Comments on "State of Charge-Dependent Polynomial Equivalent Circuit Modeling for Electrochemical Impedance Spectroscopy of Lithium-Ion Batteries"
}

\author{
Rahat Hasan and Jonathan Scott, Senior Member, IEEE
}

A recent paper by Wang et al proposes a state of chargedependent polynomial equivalent circuit model for lithium batteries. The model consists of an inductor, a Warburg element, a constant phase element (CPE) and two resistors [1]. Figure 1 shows the equivalent circuit model reproduced from [1].

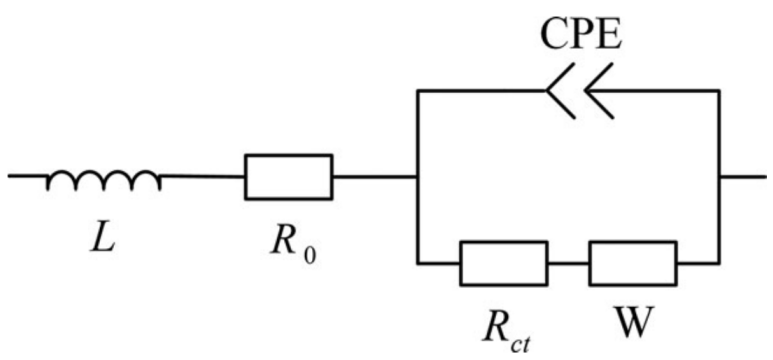

Fig. 1: Equivalent circuit model.

The authors quote the impedance equation of a Warburg element as:

$$
Z_{W}=R_{W} \frac{\tanh (j \omega \tau)^{\phi}}{(j \omega \tau)^{\phi}}
$$

where $\tau$ is the diffusion time constant, $\phi$ varies between 0 and 1 , and $R_{W}$ is the Warburg resistance. This equation is wrong; in a Nyquist plot, the angle in a Warburg element is fixed to 45 degrees at high frequencies, corresponding to $\phi=$ 0.5 . Such behaviour is a result of diffusion through a semiinfinite medium [2]. The frequency band where it is observed is called the Warburg region. The correct impedance equation of a Warburg element is of the form [3], [4]:

$$
Z_{W}=R_{W} \frac{\tanh \sqrt{(j \omega \tau)}}{\sqrt{(j \omega \tau)}}
$$

The authors cited [5] as a reference, but this paper contains no such equation.

In [1] the authors included both $\mathrm{CPE}$ and Warburg equations. The angle of the Warburg element, $\phi$, was used as an independent variable to fit the measured data. Thus an extra, unjustified degree of freedom has been created. The authors managed to fit the model to their impedance spectra with an average mean absolute percentage error of $1.04 \%$. We attribute the fitting success of the model to its having a total of 8 different parameters. Almost any model can be fitted to data with reasonable accuracy given sufficient degrees of freedom. Other researchers have obtained apparently 'good fit' where the accuracy is within $0.5 \%$ with quite different models, with a similar, excessive, number of parameters [6]. We refer readers to [7], [8] where it is shown that a single CPE and resistor model with a total of 4 degrees of freedom is capable of reproducing the impedance and charge-voltage characteristics of a lithium battery. The prediction was extraordinary with an accuracy of $0.5 \%$.

\section{REFERENCES}

[1] Qian-Kun Wang, Yi-Jun He, Jia-Ni Shen, Xiao-Song Hu, Zi-Feng Ma, "State of Charge-Dependent Polynomial Equivalent Circuit Modeling for Electrochemical Impedance Spectroscopy of Lithium-Ion Batteries", IEEE Transactions on Power Electronics, Volume: 33 , Issue: 10, 2018, pp. 8449 - 8460.

[2] Schonleber, M., Uhlmann, C., Braun, P., Weber, A., \& Ivers-Tiffee, E., "A Consistent Derivation of the Impedance of a Lithium-Ion Battery Electrode and its Dependency on the State-of-Charge", Electrochimica Acta, 2017, vol. 243, pp. 250-259.

[3] Subrata Sarker, A. J. Saleh Ahammad, Hyun Woo Seo, and Dong Min Kim, "Electrochemical Impedance Spectra of Dye-Sensitized Solar Cells: Fundamentals and Spreadsheet Calculation", International Journal of Photoenergy, Volume 2014, Article ID 851705, pages 17.

[4] H. Kahlert, U. Retter, H. Lohse, K. Siegler, and F. Scholz, "On the Determination of the Diffusion Coefficients of Electrons and of Potassium Ions in Copper(II) Hexacyanoferrate(II) Composite Electrodes", J. Phys. Chem. B, vol. 102, 1998, pp. 8757-8765.

[5] M. Doyle, J. P. Meyers, and J. Newman, "Computer simulations of the impedance response of lithium rechargeable batteries", J. Electrochem. Soc., vol. 147, no. 1, pp. 99-110, 2000.

[6] Yan Ma, Xiuwen Zhou, Bingsi Li, and Hong Chen, "Fractional Modeling and SOC Estimation of Lithium-ion Battery", IEEE/CAA JOURNAL OF AUTOMATICA SINICA, Vol.3, No. 3, July 2016, pp. 281-287.

[7] Hasan, R., and Scott, J. B., "Fractional behaviour of rechargeable batteries", Proceedings of the 2016 Electronics New Zealand Conference, November 17-18, 2016, Wellington, New Zealand, pp. 111-114.

[8] Hasan, R., and Scott, J. B., "Measurement for fractional characteristic of Lithium batteries", 2019 IEEE International Instrumentation and Measurement Technology Conference, May 20-23, 2019, Auckland, New Zealand, accepted. 